\title{
DUNCAN Y DALCROZE EN EL SISTEMA DE STANISLAVSKI: HUELLAS DE LA DANZA Y DE LA RÍTMICA
}

\author{
Virginia Analía Soprano Manzo \\ Instituto Municipal de Cultura y Juventud de Burjassot (Valencia) \\ Asociación Española Danza más Investigación
}

\begin{abstract}
Resumen
Este trabajo quiere mostrar la importancia de las técnicas corporales en el sistema para la formación del actor creado por Konstantin Stanislavski, rastreando la influencia de la danza de Isadora Duncan y la rítmica de Émile Jaques-Dalcroze en la elaboración de sus teorías. Mediante un estudio de los textos originales de Stanislavski y de sus traducciones al castellano publicadas, se constata la presencia de la danza y la rítmica en la serie de enseñanzas que componen su sistema. El nombre de Dalcroze, escrito en el texto original ruso, fue omitido en la traducción al castellano más difundida de uno de sus libros, traducida del inglés; e Isadora Duncan fue excluida en uno de sus capítulos. Omisiones, olvidos, tergiversaciones, dejaron su huella en la transmisión de un sistema que marcó el curso de la pedagogía teatral desde principios del siglo XX.
\end{abstract}

Palabras clave: STANISLAVSKI, KONSTANTIN; DUNCAN, ISADORA; JAQUES-DALCROZE, ÉMILE; SISTEMA DE STANISLAVSKI; DANZA; RÍTMICA

Abstract

This article wants to show the importance of the corporal techniques in the system for the training of actors, created by Konstantin Stanislavski, looking for the influence of dance of Isadora Duncan's and the eurhythmics of Émile Jaques-Dalcroze in the elaboration of his theories. By means of a study of the original texts of Stanislavski and of his published translations into Spanish, the presence of dance and eurhythmics is verified in the series of teachings that his system is made up of. Dalcroze's name, written in the original Russian text, was omitted in the most published Spanish translation of one of his books, translated from English; and Isadora Duncan was excluded in one of the chapters. Omissions, oversights, misrepresentations, left their mark on the transmission of a system that marked the course of theatrical teaching from the beginning of the 20th century.

Key words: STANISLAVSKI, KONSTANTIN; DUNCAN, ISADORA; JAQUES-DALCROZE, ÉMILE; STANISLAVSKI'S SYSTEM; DANCE; EURHYTHMICS

\footnotetext{
Soprano Manzo, Virginia Analía. 2015. "Duncan y Dalcroze en el sistema de Stanislavski: huellas de la danza y de la rítmica". AusArt 3 (1): 257-265. DOI: 10.1387/ausart.14768
}

\section{AUSART}


Existe una opinión generalizada respecto al sistema de formación actoral creado por Konstantin Stanislavski, una idea preconcebida que lo considera como un "método", que apelaría en primer lugar a cuestiones psicológicas, dirigido directamente a aspectos emocionales o racionales. Sin embargo, es en el cuerpo del actor donde Stanislavski concentra sus investigaciones con el movimiento, el ritmo, la acción física, para abordar por vías indirectas cuestiones psíquicas del trabajo creativo del intérprete.

Uno de los factores que determinó un entendimiento parcial del sistema de Stanislavski, lo constituye la forma en que se divulgaron las distintas ediciones y traducciones de sus textos. Diferencias de tiempo entre sus publicaciones, variantes de edición de los materiales originales, preponderancia de la traducción al inglés de la editorial neoyorquina Theatre Arts Books (desde la cual se tradujo al castellano la obra de Stanislavski dedicada a su sistema para el trabajo del actor), escasez de traducciones realizadas a partir de los textos originales rusos... Omisiones, olvidos, tergiversaciones, fueron entretejiendo una trama que confunde las enseñanzas de Stanislavski, una red cuyos nudos podemos palpar cuando, en la clase de interpretación, al joven estudiante le proponen que se emocione pensando en sus recuerdos más íntimos...

Konstantin Stanislavski escribe en sus libros sobre la danza de Isadora Duncan y la rítmica de Émile Jaques-Dalcroze. Pero el nombre de Dalcroze fue omitido en la traducción al castellano más difundida en los países de habla hispana, traducida del inglés, del libro en el que Stanislavski desarrolla las técnicas corporales exploradas en su sistema; mientras el nombre de Isadora Duncan fue excluido, en esa misma traducción, en un capítulo que trata de la visión de Stanislavski de la danza como herramienta para el desarrollo de la expresividad corporal del actor.

El objetivo del presente trabajo es dar a conocer el contenido de fragmentos de textos originales de StanisIvaski y rastrear la influencia de la danza de Isadora Duncan y la rítmica de Émile Jaques-Dalcroze en la elaboración de su sistema, para destacar el papel relevante que desempeñan el ritmo, el movimiento y la danza en la serie de enseñanzas recopiladas en sus textos.

La base metodológica de esta investigación es el estudio de los textos originales de Stanislavski, la comparación con sus traducciones al castellano publicadas y el análisis de las circunstancias de edición, publicación y traducción de sus textos. El objeto de estudio se centra en la edición rusa de su libro El trabajo del actor sobre sí mismo en el proceso creador de la encarnación. 


\section{LOS LIBROS DE STANISLAVSKI: EDICIONES, PUBLICACIONES, TRADUCCIONES}

Konstantin Sergueievitch Stanislavski, actor, director teatral y pedagogo, nacido en Moscú en 1863, publicó solo dos libros en el transcurso de su vida: una autobiografía artística, Mi vida en el arte ${ }^{1}$ y la primera parte de un libro sobre su sistema de interpretación: El trabajo del actor sobre sí mismo en el proceso creador de las vivencias ${ }^{2}$.

La segunda parte de este libro, El trabajo del actor sobre sí mismo en el proceso creador de la encarnación, fue publicada por primera vez, aunque parcialmente, en 1946, en el Anuario del Teatro de Arte de Moscú, ocho años después de la muerte de Stanislavski. En 1948 se publicó otro volumen, preparado por un comité editorial que ordenó los materiales que componen este libro: textos mecanografiados y manuscritos que el autor no había acabado de definir para su publicación. Y en 1955 se publicó en Moscú una edición corregida y ampliada, como tercer volumen de sus obras completas ${ }^{3}$.

Casi al mismo tiempo apareció en Nueva York una traducción de los mismos materiales de este libro, en una edición elaborada por Elizabeth Reynolds Hapgood, publicada por Theatre Arts Books en 1949, bajo el título Building a Character, que difiere del título propuesto por Stanislavski, el cual sugiere un proceso fluido de "encarnación". A partir de esta edición norteamericana se tradujo al castellano La construcción del personaje, obra publicada en España por primera vez en 1975, por la editorial Alianza, reeditada y reimpresa ya diecisiete veces.

Traducido directamente de los originales rusos, este libro fue publicado por primera vez en Argentina en 1977 por la editorial Quetzal, siguiendo la edición rusa de 1955; y en España, por la editorial Alba en el año 2009, a partir de una edición rusa diferente, de 1951. Ambas obras conservan el título del original: El trabajo del actor sobre sí mismo en el proceso creador de la encarnación, aunque presentan diferencias de estructura y traducción.

Lejos de constituir una cuestión puramente editorial, estas circunstancias tienen repercusiones que atañen a la difusión, interpretación y aplicación del sistema de Stanislavski. Un sistema que no es tal ya que, según Stanislavski: "No existe ningún sistema. Existe la naturaleza" ([1955] 2010, 401). Un sistema que 
explora las leyes desconocidas del cuerpo y del espíritu para restablecerlas y lograr acceder a la "naturaleza creadora", esa inconmensurable y vislumbrada región.

\section{EL SISTEMA DE STANISLAVSKI: RECIPROCIDAD ENTRE CUERPO Y PSIQUIS}

Hacia finales del siglo XIX, en una época en que los artistas dramáticos carecían de sistematización para su formación, el joven Konstantin Stanislavski, que aún intentaba la composición de personajes a partir de la imitación de modelos, ensayando frente al espejo o copiando poses de fotografías de cantantes de ópera, según él mismo relata ([1954] 1993, 105-8), fue pionero al iniciar un camino de exploración dentro del alma del artista en busca de una verdad que permitiera la fusión entre su acción interior y exterior.

El maestro ruso escribe, aludiendo al instante fugaz en que surge la musicalidad de la palabra: "¡Misterio de la naturaleza! (...) Solo ella sabe usar el aparato humano como un genial virtuoso su instrumento musical" ([1954] 2010, 418). Y dedica su esfuerzo y su trabajo al estudio de la "naturaleza creadora", no para crear en su lugar, sino para encontrar rodeos, caminos indirectos hacia ella, aquello que denomina "señuelos" ([1955] 2010, 412-13).

En el libro que estamos estudiando, El trabajo del actor sobre sí mismo en el proceso creador de la encarnación, hay un capítulo denominado "Tempo-ritmo" en el cual, mediante experimentos rítmicos con metrónomos, campanillas, palmadas, golpes de los pies en el suelo y otras percusiones, los actores van creando movimientos a partir de pautas rítmicas externas o, de modo inverso, desde su propio tempo-ritmo interior. Stanislavski descubre que, a diferencia de los otros elementos por él descubiertos que actuaban como "señuelos", el tempo-ritmo externo puede ejercer una influencia directa, muchas veces mecánica, sobre el "caprichoso, arbitrario, desobediente y asustadizo sentimiento"; y siendo así, un tempo-ritmo correctamente extraído de la obra o del rol puede hacer surgir intuitivamente la vivencia exacta en el intérprete ([1955] 2010, 239). 
Es una paradoja: en el sistema de Stanislavski, lo externo actúa sobre lo interno, y viceversa. Su contemporáneo Émile Jaques-Dalcroze, creador de la rítmica, un sistema de educación musical basado en la vivencia corporal de la música a través del movimiento, profundiza en las relaciones ritmo-cuerpo, ritmo-espíritu, sabiendo que en esta dualidad "cuerpo-alma, físico-psíquico" está la clave de toda su obra (Dutoit-Carlier 1965, 346).

\section{STANISLAVSKI, DUNCAN Y DALCROZE}

En la edición rusa consultada de El trabajo del actor sobre sí mismo en el proceso creador de la encarnación hay un capítulo titulado "Plástica", donde leemos: "A partir de hoy se introduce la clase de plástica que conduce Xenia Petrovna Sonova paralelamente a la gimnasia rítmica de Dalcroze ${ }^{4 "}$ ([1955] 2010 , 48). Dicha profesora da a los actores una pauta para generar movimientos fluidos, atrayendo su atención al movimiento de la energía a través de los músculos. A continuación, dividen esos movimientos en fracciones cada vez más pequeñas siguiendo el tempo de un metrónomo y logran una fluidez aún mayor. Stanislavski anota que "la atención física juega un importante rol (...) puesto que ayuda en la creación de la línea ininterrumpida de movimiento, tan indispensable en el arte" ([1955] 2010, 52). Y más adelante: "A estas sensaciones interiores que llevan la energía a través del cuerpo, las denominamos "sentido del movimiento" ([1955] 2010, 59).

El capítulo continúa con un análisis de la función de las distintas articulaciones del cuerpo en la acción de caminar, buscando "un modo de andar escénico, basado en las leyes de la naturaleza". Stanislavski menciona tres tipos de pasos, según la manera de utilizar las plantas y los dedos de los pies: en el primero se apoya primero el talón; en el segundo, el paso se da con la planta entera; en el tercero, denominado "paso griego à la Isadora Duncan", se apoyan primero los dedos ([1955] 2010, 59-65).

Émile Jaques-Dalcroze, músico, compositor y pedagogo suizo por adopción, nacido en Viena en 1865, también estudia el movimiento de marcha: describe cinco clases de pasos según su tamaño, velocidad y grado de resistencia muscular, y analiza la forma en que se apoyan las distintas partes del pie durante el paso $(1916,11-14)$. En el primer volumen de su método explica que su rítmica 
se basa en experiencias corporales y análisis de sensaciones físicas, y destaca la importancia de enseñar a los alumnos ejercicios de técnica corporal $(1916,7)$.

Jaques-Dalcroze da sus primeras clases experimentales de rítmica hacia 1903 en Ginebra. A partir de 1905 comienza a viajar con sus discípulos dando cursos, conferencias y demostraciones de su método. "Colocando la cuestión del cuerpo en movimiento en el centro de los debates artísticos, la rítmica ejerce en Rusia una influencia preponderante, tanto en los dominios de la puesta en escena como en la coreografía" (Suquet, 2012, 180). En una gira por Rusia, Jaques-Dalcroze visita el Teatro de Arte de Stanislavski, invitado por el príncipe Serguei Volkonski, antiguo director de los teatros imperiales, que introdujo las teorías de Dalcroze en Rusia traduciendo sus textos y fundando institutos de rítmica. Y Stanislavski asiste en 1913 al festival de Hellerau, organizado por Jaques-Dalcroze y Adolphe Appia.

Los mismos conceptos empleados por Stanislavski, citados anteriormente: "línea ininterrumpida de movimiento", "sentido del movimiento", "plástica"... fueron desarrollados por el creador de la rítmica en sus textos. Para Jaques-Dalcroze, un "movimiento continuo" debe estar presente en toda manifestación vital animada por un "pensamiento continuo", y una "serie ininterrumpida de movimientos” constituye lo que denomina "melodía plástica” (1920, 167-68).

Pero el nombre de Dalcroze está ausente en la traducción del libro de Stanislavski publicada por la editorial Alianza, La construcción del personaje, en el capítulo que es traducido "Plasticidad del movimiento", donde leemos: "Paralelamente a nuestros ejercicios de gimnasia rítmica hemos comenzado un curso de movimiento plástico a cargo de Madame Sonova" ([1949] 2010, 73). Tampoco lo encontramos en el capítulo "Plástica" de la traducción de la editorial Alba: "Hoy han comenzado las clases de plástica, que imparte Xenia Petrovna Sonova, paralelamente a las de gimnasia rítmica" ([1951] 2009, 77). Sí está presente el nombre de Dalcroze en el capítulo "Plasticidad de los movimientos" de la traducción argentina de la editorial Quetzal, agotada y reimpresa recientemente: "Paralelamente con nuestras prácticas de gimnasia rítmica con Dalcroze comenzamos hoy los ejercicios de movimientos plásticos, bajo la dirección de Xenia Petrovna Sonova" ([1955] 1986, 42).

"Plástica" es un concepto que utiliza Dalcroze. Para el maestro suizo, "el movimiento corporal es una experiencia muscular (...) apreciada por un sexto sentido que es el sentido muscular" $(1920,168)$. Este sentido es el que permite 
transformar en movimientos la percepción de los elementos musicales, lo que Jaques-Dalcroze llamó "plástica animada" y después simplemente "plástica".

"Plástica" es además el término con el que se designan en Rusia las formas derivadas de la danza libre de Isadora Duncan. Bailarina y pedagoga revolucionaria, nacida en San Francisco en 1878, Isadora hizo sus primeras apariciones en San Petersburgo y Moscú en 1904 y 1905. Las repercusiones de su danza en Rusia fueron profundas. Muchos artistas experimentaron una revelación al verla bailar, pies desnudos. También Stanislavski, desde la primera vez que presenció una de sus actuaciones, descubrió en ella una profunda afinidad artística.

En el libro El trabajo del actor sobre sí mismo en el proceso creador de la encarnación hay otro capítulo, titulado "Gimnasia, acrobacia, danzas, etc.", donde Stanislavski expone su opinión de la danza como herramienta para el entrenamiento del actor, expresando: "No me gusta la plástica de las manos en las bailarinas. Resulta amanerada, condicionada y sentimental (...). Muchas bailarinas danzan con muñecas y dedos muertos, estáticos, forzados. Esta vez sería mejor que recurriéramos a la ayuda de la escuela de Isadora Duncan. Allí se dominan mejor las muñecas" ([1955] 2010, 43).

El nombre de Isadora Duncan aparece dos veces en la edición rusa de este libro, en el capítulo mencionado en el párrafo anterior y en el capítulo "Plástica" anteriormente citado, al nombrar el "paso à la Isadora Duncan". Tanto en la edición argentina de la editorial Quetzal, como en la española Alba, aparece también dos veces, en los mismos lugares. Sin embargo, en la traducción de la editorial Alianza, La construcción del personaje, el nombre de la bailarina norteamericana solo aparece la segunda vez, pero no la primera, allí donde Stanislavski habla de su opinión de la danza porque está buscando una plástica corporal que pueda adaptarse al arte del actor.

Empezar a desanudar esa trama hecha de omisiones, olvidos y tergiversaciones en los textos de Stanislavski conociendo las fuentes, mediante una lectura despojada de prejuicios, permite descubrir las huellas que dejaron la danza de Isadora Duncan y la rítmica de Émile Jaques-Dacroze en las enseñanzas de un sistema de interpretación que explora el cuerpo como un espacio donde pueden sintetizarse técnicas provenientes no solo del teatro, también de la música y la danza. 


\section{Referencias}

Dutoit-Carlier, Claire-Lise. 1965. Émile Jaques-Dalcroze, L'homme, le compositeur, le créateur de la rythmique. Neuchâtel: La Baconnière

Jaques-Dalcroze, Émile. 1916. La rythmique: enseignement pour le développement de l'instinct rythmique et métrique, du sens de l'harmonie plastique et de l'equilibre des mouvements, et pour la régularisation des habitudes motrices. Vol. 1. Lausana: Jobin

- 1920. Le Rythme, la Musique et l'Éducation. París: Fischbacher \& Rouart; Lausana: Jobin

Stanislavski, Konstantin. (1949) 2010. La construcción del personaje. Traducido por Bernardo Fernández. Madrid: Alianza

- (1951) 2009. El trabajo del actor sobre sí mismo en el proceso creador de la encarnación. Traducido por Jorge Saura. Barcelona: Alba

- (1954) 2010. Моя жизнь в искусстве. [Mi vida en el arte]. Moscú: Издательство АСТ Зебра $\mathrm{E}$

- (1954) 1993. Mi vida en el arte. Traducido por Salomón Merener. Buenos Aires: Quetzal

- (1954) 1977. El trabajo del actor sobre sí mismo en el proceso creador de las vivencias. Traducido por Salomón Merener. Buenos Aires: Quetzal

- (1955) 2010. Работа актёра над собой. Работа актёра над собой в творческом процессе воплощения. [El trabajo del actor sobre sí mismo. El trabajo del actor sobre sí mismo en el proceso creador de la encarnación]. San Petersburgo: Прайм-Еврознак

- (1955) 1986. El trabajo del actor sobre sí mismo en el proceso creador de la encarnación. Traducido por Salomón Merener. Buenos Aires: Quetzal

Suquet, Annie. 2012. L'Éveil des modernités: Une histoire culturelle de la danse (1870-1945). Pantin: Centre National de la Danse

Notas

${ }^{1}$ La primera edición rusa de Моя жизнь в искусстве (Mi vida en el arte) fue publicada en 1926, la segunda en 1928, y la tercera en 1954 como primer volumen de Собрание сочинений (Obras completas). En 1924, la editorial americana Little-Brown and Co. ya había publicado en Boston My Life in Art, obra escrita por Stanislavski después de una exitosa gira por Estados Unidos y que fue reelaborada por él mismo para la edición rusa.

${ }^{2}$ Esta primera parte de El trabajo del actor sobre sí mismo fue publicada por primera vez en la Unión Soviética en 1938, y en 1954 como segundo tomo de Собрание сочинений (Obras completas) de Stanislavski. En 1936 la editorial Theatre Arts Books había publicado en Nueva York An Actor Prepares, una edición elaborada por Elizabeth Reynolds Hapgood y Norman Hapgood a partir de los originales rusos de este mismo libro.

${ }^{3}$ Datos extraídos del prólogo, redactado por Grigori Vladimirovitch Kristi, de esta edición rusa de 1955 del libro de Stanislavski Работа актёра над собой. Работа актёра над собой в творческом процессе воплощения (El trabajo del actor sobre sí mismo. El trabajo del actor sobre sí mismo en el proceso creador de la encarnación), tercer tomo de Собрание сочинений (Obras completas), publicadas en Moscú por la editorial Искусство.

${ }^{4}$ Poco después del nacimiento de la rítmica, Dalcroze le dio el nombre de Gimnasia Rítmica a fin de que nadie ignorara que estaba basada en el movimiento. Creando un equívoco este primer término, no señalando suficientemente la noción de arte que la compone, 
lo suprime dejando a la posteridad un nombre inseparable de su apellido: "Rítmica Jaques-Dalcroze" (Dutoit-Carlier 1965, 327-28). 